\title{
The effects of self-focus on attentional biases in social anxiety: An ERP study
}

\author{
Matt R. Judah ${ }^{1}$ • DeMond M. Grant ${ }^{1}$ • Nancy B. Carlisle ${ }^{2}$
}

Published online: 16 December 2015

(C) Psychonomic Society, Inc. 2015

\begin{abstract}
Cognitive theories of social anxiety disorder suggest that biased attention plays a key role in maintaining symptoms. These biases include self-focus and attention to socially threatening stimuli in the environment. The goal of this study was to utilize ERPs that are elicited by a change detection task to examine biases in selective attention (i.e., $\mathrm{N} 2 \mathrm{pc}$ ) and working memory maintenance (i.e., contralateral delay activity; CDA). Additionally, the effect of self-focus was examined using false heart rate feedback. In support of the manipulation, self-focus cues resulted in greater selfreported self-consciousness and task interference, enhanced anterior P2 amplitude and reduced SPN amplitude. Moreover, P2 amplitude for self-focus cues was correlated with reduced task performance for socially anxious subjects only. The difference in P2 amplitude between self-focus and standard cues was correlated with social anxiety independent of depression. As hypothesized, socially anxious participants $(n=20)$ showed early selection and maintenance of disgust faces relative to neutral faces as indicated by the N2pc and CDA components. Nonanxious controls $(n=22)$ did not show these biases. During self-focus cues, controls showed marginal evidence of biased selection for disgust faces, whereas socially anxious subjects showed no bias in this condition. Controls showed an ipsilateral delay activity after being cued to attend to one hemifield. Overall, this study supports early and persistent attentional bias for social threat in socially anxious individuals. Furthermore, self-focus may disrupt these
\end{abstract}

Matt R. Judah

matt.judah@okstate.edu

1 Department of Psychology, Oklahoma State University, Stillwater, OK 74078, USA

2 School of Psychology, University of Leicester, Leicester, UK biases. These findings and supplementary data are discussed in light of cognitive models of social anxiety disorder, recent empirical findings, and treatment.

Keywords Social anxiety $\cdot$ N2pc $\cdot$ CDA $\cdot$ Self-focus . Attentional bias

Attentional biases play a key role in cognitive models of social anxiety disorder (SAD; Clark \& McManus, 2002; Heimberg, Brozovich, \& Rapee, 2010). These biases include (1) focusing on how features of the self, especially anxiety symptoms, appear to others (i.e., self-focus) and (2) attention to external cues of possible rejection. Thus, internal anxiety symptoms (e.g., increased heart rate) and external signals of potential social threat (commonly operationalized as disgust faces; Amir et al., 2005; Amir, Najmi, Bomyea, \& Burns, 2010; Buckner, Maner, \& Schmidt, 2010; Judah, Grant, Lechner, \& Mills, 2013) are thought to be prioritized by selective attention and maintained in working memory. External social threat biases have been supported by a variety of methodologies (see Staugaard, 2010), including emotional Stroop (Amir, Freshman, \& Foa, 2002; Grant \& Beck, 2006), dot-probe (Mogg, Philippot, \& Bradley, 2004; Sposari \& Rapee, 2007), and visual search tasks (Gilboa-Schechtman, Foa, \& Amir, 1999; Juth, Lundqvist, Karlsson, \& Öhman, 2005). However, less research has examined self-focus or its effect on external biases.

Cognitive theories of SAD propose that self-focus involves observer perspective images of the self in order to assess the likelihood of negative evaluation. This is especially the case for aspects of the self that are believed to be seen by others as negative, such as somatic anxiety symptoms. Although sensitivity to somatic symptoms is a common feature of anxiety pathology, especially panic disorder (see Domschke, Stevens, 
Pfleiderer, \& Gerlach, 2010), it has a unique function in SAD because it prompts fears of rejection if others notice these symptoms (Spurr \& Stopa, 2002). In support of this, research suggests that individuals with SAD monitor their physiology, particularly their heart rate, and this prompts the formation of negative self-images (Mansell, Clark, \& Ehlers, 2003; Papageorgiou \& Wells, 2002) and negatively biased beliefs about evaluation (Mansell \& Clark, 1999; Mellings \& Alden, 2000). Socially anxious individuals also show attentional bias for heart-rate images relative to images of sound waves after imagining an upcoming social interaction (Mills, Grant, Judah, \& White, 2014). Several studies have used pseudo heart-rate feedback to manipulate self-focused attention. These studies have found that it increases self-focused attention, self-reported anxiety, negative ratings of social performance, task interference, and observer perspective anticipation of social interactions for socially anxious subjects (Judah, Grant, Mills, \& Lechner, 2013; Mansell et al., 2003; Papageorgiou \& Wells, 2002; Wells \& Papageorgiou, 2001; Wild et al., 2008). For example, Judah and colleagues (Judah, Grant, Mills, et al., 2013) told participants that a fixation symbol would change when their heart rate accelerated, though the fixation symbol changed randomly in reality. They found that this reduced the amplitude of the stimulus-preceding negativity (SPN), an event-related potential (ERP) component associated with preparation for an upcoming stimulus or response.

With respect to working memory, research suggests that social anxiety may be associated with difficulty filtering nonemotional task-irrelevant information from visual working memory (Moriya \& Sugiura, 2012a), particularly for those with low working memory capacity (Moriya \& Sugiura, 2013) and regardless of whether cognitive load is low or high (Moriya \& Sugiura, 2012b). A few studies have examined biases for socially threatening stimuli in social anxiety. One study found that subjects with SAD displayed greater working memory capacity compared to healthy controls for social threat words but not neutral words (Amir \& Bomyea, 2011). Another found that socially anxious individuals may be better at preventing storage of task-irrelevant positive information in working memory (Segal, Kessler, \& Anholt, 2015).

Despite this evidence, several theoretical and empirical discrepancies persist. Whereas one perspective proposes that self-focused attention preoccupies cognitive resources, thereby reducing attention to social cues (Clark \& McManus, 2002), another suggests that it may increase biases for social threat (Heimberg et al., 2010). Additionally, the literature is replete with mixed findings regarding external attentional biases, especially after initial detection of threat. Whereas many studies suggest that socially anxious subjects direct attention away from socially threatening stimuli after detecting them (e.g., Mogg et al., 2004; Singh, Capozzoli, Dodd, \& Hope, 2015), other studies suggest that they show difficulty disengaging attention from social threat (e.g., Buckner et al., 2010; Schofield et al., 2012).

In order to resolve these discrepancies, researchers have evaluated moderators of these equivocal results. For example, Judah and colleagues (Judah, Grant, Lechner, et al., 2013) used dot-probe methodology with a secondary $n$-back task to test whether cognitive load interfered with the ability to disengage attention from social threat. Supporting this hypothesis, they found evidence of avoidance of disgust faces for trials without load or with low levels of load, but bias toward threat when cognitive load was high. This study identified cognitive load as an important moderator of late attentional bias by observing difficulty disengaging attention from social threat only when load was present. Although such studies may advance our understanding of attentional biases in social anxiety, they have notable limitations. Specifically, reaction time tasks measure attention indirectly and include variance from nonattentional processes. Therefore, more research is needed which (1) assesses attentional biases with greater temporal precision (2) and uses direct measures of attentional deployment.

ERPs, a noninvasive measure of summated cortical postsynaptic potentials, are particularly well-suited for examining attentional biases. ERPs can provide a temporally precise, direct measure of covert attention and may detect biases not evident in behavioral data (see Kappenman, Farrens, Luck, \& Proudfit, 2014). Several studies using ERPs and related approaches have suggested that socially anxious individuals display enhanced attention to emotional faces (McTeague, Shumen, Wieser, Lang, \& Keil, 2011; Rossignol, Campanella, et al., 2012; Rossignol, Philippot, Bissot, Rigoulot, \& Campanella, 2012), especially those which are aversive (Kolassa, Kolassa, Musial, \& Miltner, 2007; Moser, Huppert, Duval, \& Simons, 2008; Mueller et al., 2009; Mühlberger et al., 2009; Sewell, Palermo, Atkinson, \& McArthur, 2008). For example, studies have found evidence of enhanced attention for angry faces in social anxiety as indicated by the P2 (Rossignol, Campanella, Bissot, \& Philippot, 2013; Van Peer, Spinhoven, \& Roelofs, 2010), although other studies suggest enhanced attention for faces more generally (Rossignol, Philippot, Bissot, Rigoulot, \& Campanella, 2012) or not at all (Kolassa et al., 2009). Many other studies have examined the P1, which may be larger in socially anxious individuals more generally due to enhanced task engagement (see Peschard, Philippot, Joassin, \& Rossignol, 2013). Research using lateralized ERPs may advance this literature because these components allow comparison of attention to stimuli presented simultaneously within each side of the visual field. Furthermore, they may advance this 
literature by enabling researchers to examine the persistence of biases in working memory.

Two lateralized ERPs, the N2pc and contralateral delay activity (CDA), are well-established as useful for examining selective attention and maintenance in visual working memory, respectively. Both ERPs are evident at posterior-occipital scalp sites contralateral to the side of the visual field that is preferentially attended. The N2pc emerges about $200 \mathrm{~ms}$ after stimulus presentation and has been used to examine selective attention for objects in one hemifield (i.e., side of the visual field) relative to those in the other (e.g., Luck \& Hillyard, 1994; Weymar, Gerdes, Löw, Alpers, \& Hamm, 2013), including facial expressions (Eimer \& Kiss, 2007). The CDA emerges about $300 \mathrm{~ms}$ after a typically brief (e.g., $100 \mathrm{~ms}$ ) stimulus presentation, and the CDA persists as the stimulus is maintained in working memory (Luck \& Hillyard, 1994; Vogel \& Machizawa, 2004). Typically, the CDA is elicited using a change detection paradigm (see Vogel, McCollough, $\&$ Machizawa, 2005). Bilateral visual stimulus arrays are presented briefly after a target cue that indicates which side of the stimulus array should be attended. After a retention interval, a test array is presented, and subjects are required to report whether or not any stimulus within the target hemifield has changed. The CDA has been used to examine biases in visual working memory for fearful facial expressions in anxious individuals (Stout, Shackman, \& Larson, 2013).

The goal of this study was to examine (1) whether socially anxious individuals display biases in selection and working memory storage for socially threatening faces and (2) how self-focused attention affects these biases. This was examined using a modified change detection task with disgust and neutral faces. A cue to attend to one side (i.e., target cue) was presented after this array to allow us to assess biases when trying to control attention based on task demands (after target cue) and when uncontrolled (before target cue). Self-focused attention was manipulated for $50 \%$ of trials by providing a randomly appearing change in the fixation symbol (i.e., selffocus cue), which participants were told beforehand would indicate elevated heart rate.

We hypothesized that socially anxious individuals would display biased selection (i.e., greater N2pc amplitude) and working memory maintenance (i.e., greater CDA amplitude) for socially threatening facial expressions, as predicted by cognitive models of SAD. We hypothesized that these biases would persist after the task cue, enhancing maintenance (CDA) of disgust targets and reducing maintenance for neutral targets, indicating difficulty filtering task-irrelevant disgust faces from working memory. We expected that self-focus might enhance these effects (Judah, Grant, Mills, et al., 2013; Rapee \& Heimberg, 1997), although other theoretical perspectives (e.g., Clark \& Wells, 1995) predict reduced attention toward external stimuli, including socially threatening faces.

\section{Method}

\section{Participants}

The sample $(N=42)$ consisted of 26 female $(62 \%)$ and 16 male undergraduate students recruited using an online research participation system at a large Midwestern university (see Table 1 for demographics). The Social Interaction Anxiety Inventory (SIAS; Mattick \& Clarke, 1989) was used to sort the sample into social anxiety groups. Cutoffs were selected based on the mean (i.e., 19) and standard deviation (i.e., 10) of the undergraduate sample in the original SIAS study (Mattick \& Clarke, 1989). As in other studies (e.g., Judah, Grant, Lechner, et al., 2013; Judah, Grant, Mills, et al., 2013), subjects scoring more than 1 standard deviation above this mean (i.e., $\geq 30$ ) constituted the high social anxiety group (HSA; $n=20$ ), and those scoring at or below the mean (i.e., $\leq 19)$ made up the low social anxiety group (LSA; $n=$ 22). An a priori power analysis using effect sizes from a similar study (Stout et al., 2013) and Cohen's (1988) procedures suggested that 15 to 18 subjects were needed for each group to have power $\geq .80$.

\section{Measures}

Demographics form A brief questionnaire was used to obtain information about each participant's sex, race, education, and other pertinent demographic data.

Social interaction anxiety scale (SIAS; Mattick \& Clarke, 1989). This is a 20 -item self-report measure of fears related to social interactions, with higher scores reflecting higher levels of social anxiety. Internal consistency was excellent for this study (Cronbach's $\alpha=.94$ ).

Beck depression inventory (BDI-II; Beck, Steer, \& Brown, 1996). The BDI-II was used as a control variable in follow-up analyses because depression and social anxiety symptoms are highly comorbid (see Pini et al., 1997) and research suggests that attentional biases linked to social anxiety may differ in those who are also depressed (see LeMoult \& Joormann, 2012). This is a 21 -item measure of self-reported depression symptoms during the most recent 2 weeks. Studies support the reliability and validity of the BDI-II (e.g., Storch, Roberti, \& Roth, 2004), and internal consistency was high in this study (Cronbach's $\alpha=.85$ ).

The Wechsler adult intelligence scale III, letter-number sequencing subtest (Wechsler, 1997). This task was administered to compare working memory capacity between groups and to rule it out as a potential explanation for observed effects. It involves hearing lists of letters and numbers and reciting the numbers first in numerical order followed by the 
Table 1 Group Comparisons

\begin{tabular}{|c|c|c|c|c|c|c|}
\hline \multirow[b]{2}{*}{ Variables } & \multicolumn{2}{|c|}{ HSA Group } & \multicolumn{2}{|l|}{ LSA Group } & \multicolumn{2}{|c|}{ Significance Test } \\
\hline & $\begin{array}{l}M \\
\text { (Count) }\end{array}$ & $\begin{array}{l}S D \\
(\%)\end{array}$ & $M$ (Count) & $\begin{array}{l}S D \\
(\%)\end{array}$ & $\begin{array}{l}t \\
\left(\chi^{2}\right)\end{array}$ & $p$ \\
\hline Sex & - & - & - & - & $(.77)$ & .38 \\
\hline Male & (9) & $(45 \%)$ & (7) & $(32 \%)$ & & \\
\hline Female & (11) & $(55 \%)$ & $(15)$ & $(68 \%)$ & & \\
\hline Age & 19.80 & 5.34 & 18.95 & 1.81 & 1.91 & .18 \\
\hline Ethnicity & - & - & - & - & $(4.95)$ & .29 \\
\hline Caucasian & (18) & $(90.0)$ & (17) & $(77.3)$ & & \\
\hline African American & $(0)$ & $(0)$ & (3) & $(14.6)$ & & \\
\hline Native American & $(0)$ & $(0)$ & (1) & $(5.5)$ & & \\
\hline Biracial & (1) & $(5.0)$ & $(0)$ & $(0)$ & & \\
\hline No response & (1) & $(5.0)$ & (1) & $(5.5)$ & & \\
\hline Letter-number seq. & 11.31 & 1.93 & 11.02 & 2.57 & .56 & .58 \\
\hline Performance & .53 & .05 & .55 & .05 & 1.29 & .20 \\
\hline Response rate & .91 & .08 & .92 & .06 & .36 & .73 \\
\hline Self-focus cue & - & - & - & - & - & - \\
\hline Self-consciousness & 3.40 & 1.70 & 2.59 & 1.84 & 1.48 & .15 \\
\hline Interference & 2.95 & 1.88 & 2.45 & 2.09 & .81 & .43 \\
\hline
\end{tabular}

letters in alphabetical order. Participants completed three trials of each sequence set size, which ranged from two to eight. A total score was computed as the number of correct trials, with a possible score range of 0 to 21 . Following the standard procedure of the Letter-Number Sequencing task, participants continued with increasing set sizes until they responded incorrectly on all three trials within a set size.

The radboud faces database (Langner et al., 2010). This is a freely available online collection of facial stimuli that has been standardized and validated for use in cognitive studies. Disgust and neutral faces from a frontal view with direct eye gaze were used in this study. Studies suggest that socially anxious individuals perceive disgust faces as more threatening than other emotional faces (Amir et al., 2005; Amir et al., 2010). Sixteen male and 16 female adult Caucasian faces were selected for this study. In order to remove irrelevant features (i.e., neck, shoulders, and space around the head) and maximize the size of facial stimuli, each stimulus was cropped to a rectangle with a $.73: 1$ width-to-height ratio.

\section{Procedure}

All procedures were approved by the Institutional Review Board. Following informed consent, participants completed self-report measures online followed by a letter-number sequencing task and the modified change detection task (see Fig. 1). Stimulus array positioning, presentation times, and other features of the change detection task were modeled after similar studies (e.g., Stout et al., 2013). Participants completed 1,024 trials to ensure that at least 250 trials per condition were presented and that stimuli were balanced. Eight blocks consisting of 128 trials were administered with 20 -second breaks following Trials 42 and 84 within each block and breaks of at least 60 seconds between blocks (see Woodman \& Vogel, 2008). Participants were instructed before the task that it would be difficult and that it was important for them to do their best throughout the entire experiment. Emphasis on performance provided a social evaluative context for the heart-rate feedback in order to elicit self-focused attention as described in cognitive models.

Stimuli were presented on a Dell 19-inch LCD monitor with a refresh rate of $60 \mathrm{~Hz}$. Each subject was seated so that the nasion was $70 \mathrm{~cm}$ from the monitor. The height of the monitor was adjusted so that its center aligned with each subject's line of sight. Stimulus presentation and event logging were controlled using PsychoPy (Version 1.80; Peirce, 2007). Participants were given the following instructions prior to the task: "For this task you will see pictures of faces appearing on each side of the screen. After the faces disappear, you will be asked to remember the faces on one side of the screen. The faces will appear again, and you will indicate whether the identity of any of the faces on that side of the screen changed. Please press the (left/right trigger) if there is a change and the (left/right trigger) if there is not." Participants then completed 14 monitored practice trials with automated feedback (i.e., "Correct" or "Incorrect"). For each trial, a fixation symbol $(+)$ appeared in the center of the monitor, and participants 


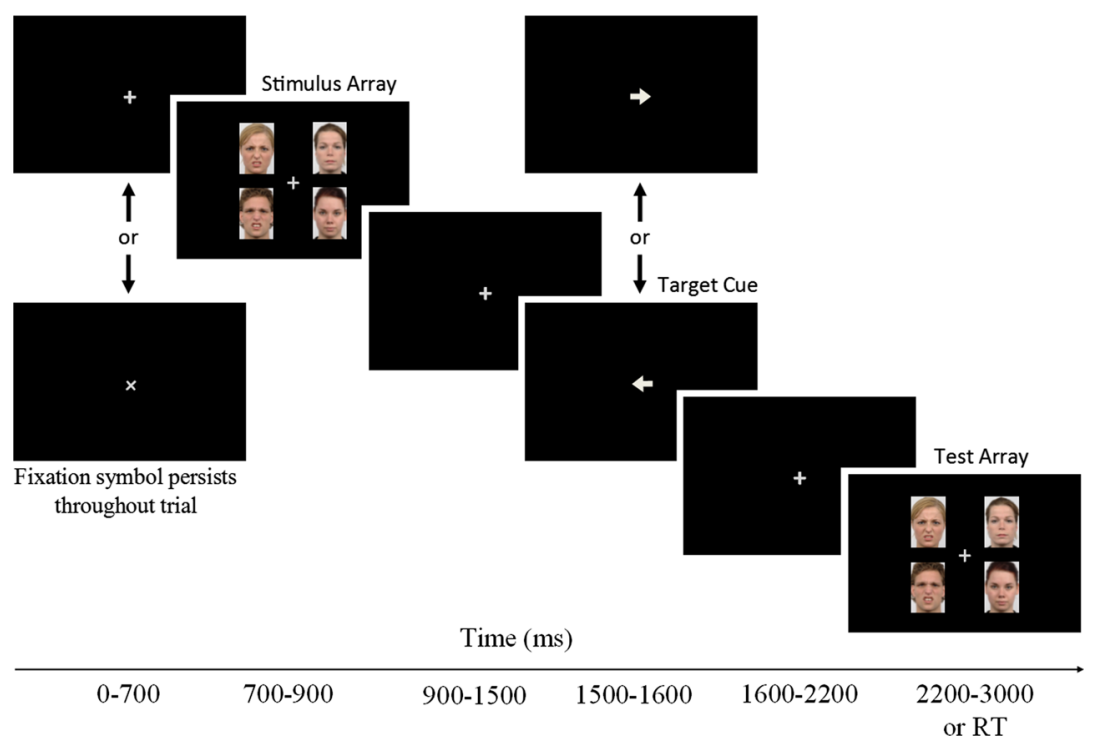

Note: N2pc and uncued CDA locked to onset of Stimulus array at $700 \mathrm{~ms}$ and measured from 900-1000 ms and 1000-1500 ms respectively. Cued CDA locked to onset of Target Cue at 1500 $\mathrm{ms}$ and measured from $1700-2200 \mathrm{~ms}$.

Fig. 1 Change Detection Task

were told to look at it throughout the study and to minimize eye movements. On $50 \%$ of trials, participants were told that an " $\times$ " would appear instead of the standard fixation symbol (i.e., + ) if their heart rate increased. To increase believability, participants were told that this was caused by a software glitch and that they could ignore it as it was not relevant to the task. The psychological meaning of these symbols was counterbalanced across subjects.

Each trial began with a centrally located fixation symbol or false heart-rate symbol presented for $700 \mathrm{~ms}$. This fixation symbol remained present throughout the remainder of the trial and retained its orientation. After this, a stimulus array consisting of two neutral face pictures arranged vertically on one side of the screen and two disgust face pictures arranged vertically on the other side of the screen was presented for 200 ms. All faces were taken from the Radboud database (Langner et al., 2010). Each face had a $2.3^{\circ}$ horizontal offset from the fixation symbol, and the visual angle between vertically arranged stimuli was $2.2^{\circ}$. The visual angle subtended by the fixation symbol was $1.2^{\circ}$, and that of each face picture was $4.7^{\circ} \times 6.5^{\circ}$. The $\mathrm{N} 2 \mathrm{pc}$ was measured from an epoch event locked to presentation of the face stimulus array. Following stimulus presentation, the fixation symbol remained without other stimuli for $600 \mathrm{~ms}$, during which time the uncued CDA was measured. After this, an arrow (i.e., target cue) pointing to the right or left (equal probability) appeared in the center of the screen for $100 \mathrm{~ms}$ followed by presentation of the fixation symbol by itself for $600 \mathrm{~ms}$, during which time the cued CDA was measured. After this, the test array appeared for $800 \mathrm{~ms}$ or until a response was made, whichever occurred first. Test arrays were identical to stimulus arrays for $50 \%$ of trials or had one face changed on the side indicated by the arrow for the other $50 \%$ of trials. Changed faces consisted of a pseudorandomly selected face that appeared on other trials. Changed faces were a different gender for $50 \%$ of trials. Throughout the task, face stimuli were presented an equal number of times in the face stimulus arrays (i.e., 128 times) and as changed stimuli in test arrays (16 times). Intertrial intervals varied randomly using latencies ranging from 600 to $900 \mathrm{~ms}$.

\section{Manipulation check}

Based on previous research, participants responded in writing to an open-ended question after the experiment about what caused the computer glitch and rated how much the selffocus cues increased their self-consciousness and interfered with their performance on a scale of 0 (not at all) to 7 (extremely; Judah, Grant, Mills, et al., 2013; Papageorgiou \& Wells, 2002). To evaluate differential attention to self-focus cues, the anterior $\mathrm{P} 2$, also known as the selection positivity, an ERP maximal at approximately $200 \mathrm{~ms}$, was examined. Research suggests that P2 amplitude is enhanced when subjects show stronger selective attention for a stimulus (AnlloVento, Luck, \& Hillyard, 1998; Potts, 2004). The effect of self-focus on anticipation of the stimulus arrays was assessed using the SPN, a fronto-centrally maximal slow-wave indicative of anticipatory attention for an impending stimulus (Van Boxtel \& Böcker, 2004). Research suggests that anxiety related cognitive processes are associated with reduced SPN amplitude (Grant et al., 2015), and a self-focus manipulation similar to that in the current study was found to reduce the 
SPN in socially anxious individuals (Judah, Grant, Mills, et al., 2013).

\section{Electrophysiological recording}

Electroencephalographic (EEG), electrocardiographic (ECG), and electrooculographic (VEOG and HEOG) data were collected using an Active II system (BioSemi, Amsterdam, The Netherlands). EEG data were collected from 32 channels (Fp1/2, AF3/4, F7/8, F3/4, FZ, FC5/6, FC1/2, T7/8, C3/4, CZ, $\mathrm{CP} 5 / 6, \mathrm{CP} 1 / 2, \mathrm{P} 7 / 8, \mathrm{P} 3 / 4, \mathrm{PZ}, \mathrm{PO} 3 / 4, \mathrm{O} 1 / 2$, and OZ) positioned using the $10 / 20$ system.

\section{Electrophysiological data processing}

Data were sampled at $256 \mathrm{~Hz}$, referenced online to the Common Mode Sense (CMS) active electrode, filtered online with a .01-100 Hz band pass filter, filtered offline with a band pass filter of .1-30 Hz (Butterworth, $12 \mathrm{~dB} /$ oct. roll-off), and referenced offline to the average of the mastoids. EEGLAB, Version 11 (Delorme \& Makeig, 2004), and ERPLAB, Version 4.0 (Lopez-Calderon \& Luck, 2013), were used to process the data. Independent components analysis (ICA) was used to correct for ocular artifact, which was identified by evaluating the spatial and temporal characteristics of each component (see Mognon, Jovicich, Bruzzone, \& Buiatti, 2011). The mean amplitude during the $200 \mathrm{~ms}$ prior to the most recent stimulus onset was subtracted to baseline each epoch. Automated routines in ERPLAB were used to detect artifacts (e.g., blinks and saccades), and trials containing artifacts at critical times were excluded from averaging. More specifically, trials containing blinks within $200 \mathrm{~ms}$ of the onset of the fixation cross, stimulus array, directional cue, or test array were excluded, as were trials containing saccades between the onset of the stimulus and test arrays. Additionally, automated routines were used to reject trials with artifacts, including large voltage changes and blocking. No more than $25 \%$ of trials were rejected for any subject. The LSA and HSA groups did not differ in number of trials retained or rejected for any trial type (all $t \mathrm{~s}<1.30, p \mathrm{~s}>.22$ ). The N2pc and CDA were evaluated by pooling electrodes $\mathrm{P} 3 / 4, \mathrm{PO} 3 / 4$, and $\mathrm{O} 1 / 2$. Electrodes were averaged with their laterally corresponding locations depending on which hemifield contained the stimulus being evaluated. The N2pc was measured as the mean amplitude within the 200 to $300 \mathrm{~ms}$ poststimulus measurement window (Kiss, Van Velzen, \& Eimer, 2008). Uncued and cued CDA amplitudes were measured as the mean amplitudes from $300 \mathrm{~ms}$ poststimulus until the onset of the next stimulus (i.e., $800 \mathrm{~ms}$ for uncued CDA, $700 \mathrm{~ms}$ for cued CDA; Vogel et al., 2005). Scalp maps representing mean amplitude within these windows verified that ERPs were maximal at the measured occipito-parietal sites.

\section{Analytic strategy}

Prior to the main analyses, the success of the self-focus manipulation was evaluated by examining anterior P2 amplitude differences for the standard and self-focus fixation symbols between groups. To examine the effect of the self-focus manipulation on anticipation of the stimulus arrays, the SPN in the $300 \mathrm{~ms}$ prior to the onset of these arrays was examined. Performance was assessed as a function of Condition (SelfFocus, Standard) and Target (Disgust, Neutral) using repeated measures ANOVA. The current study used a mixed factorial design consisting of a between-groups factor, Group (HSA, LSA), and two within-subjects factors, Condition (Self-focus, Standard) and Laterality (Contra, Ipsi), as well as three dependent variables (amplitudes of the N2pc, uncued CDA, cued CDA). Thus, three repeated-measures ANOVAs were used to evaluate the data. For the cued CDA, there was an additional within-subjects factor, Target (Disgust, Neutral). Significant interactions were probed using simple effects analysis with Bonferroni adjustments. This resulted in an adjusted $p$ critical value of .013 for follow-up comparisons.

Prior to the analyses, the data were examined for violations of the assumptions of ANOVA. There were no outliers among ERP amplitudes (i.e., all $Z$ scores < 3.29). Fmax values were below 10. Skewness and kurtosis values were within acceptable limits (i.e., less than 2.0 and 4.0, respectively) for the N2pc and uncued CDA. For the cued CDA, there was slight negative skew for self-focus trials at sites contralateral $(-2.14)$ and ipsilateral $(-2.02)$ to disgust faces. Distributions of cued CDA amplitudes were slightly leptokurtotic for self-focus trials, with the highest kurtosis value being 6.35 .

\section{Results}

\section{Sample characteristics}

There were no significant differences between the LSA and HSA groups in terms of demographic variables, task response rate, task performance, or working memory capacity as measured by letter-number sequencing (see Table 1).

\section{Manipulation check}

All subjects recalled that the change in the fixation symbol was a software glitch that indicated that their heart rate had accelerated. Subjects reported that selffocus cues resulted in a significant increase (i.e., scores $>0)$ in self-consciousness, $t=10.71, p<.001$, and interference with performance, $t=8.80, p<.001$. The HSA and LSA groups did not differ in self-reported self-consciousness, $t=1.48, p=.15$, or interference, $t$ $=.81, p=.43$, due to self-focus cues. Pooled FZ and 
$\mathrm{CZ}$ were examined within the 200 to $250 \mathrm{~ms}$ window to evaluate the anterior P2 following the onset of fixation symbols (see Fig. 2). A 2 [Group (HSA, LSA)] $\times 2$ [Condition (Standard, Self-Focus)] mixed ANOVA revealed a main effect for Condition, $F(1,40)=5.70, p$ $=.02, \eta_{\mathrm{p}}{ }^{2}=.13$, such that P2 amplitude was larger for self-focus cues $(M=1.70, S D=2.19)$ compared to standard fixation symbols $(M=1.20, S D=2.94 ; d=$ .19). The main effect for Group was not significant, $F(1,40)=2.95, n s$, nor was the interaction, $F<1.0$. Moreover, P2 amplitude for self-focus cues, but not standard cues, was negatively correlated with performance for socially anxious subjects, $r=-.49, p=.03$, suggesting that attention to self-focus cues was associated with task interference. This correlation was not significant for the low social anxiety group, $p=.20$. There was a significant partial correlation between social anxiety and the P2 difference wave (self-focus P2 minus standard P2) when controlling for depression, $r=$ $.30, p=.03$. This suggests that the portion of the P2 modulated by physiological sensitivity was correlated with social anxiety independent of depressive symptoms. These analyses supported the manipulation by suggesting that subjects displayed greater selective attention for self-focus cues than for standard fixation symbols and that social anxiety was associated with greater attention to self-focus cues.

The SPN was examined at pooled sites FZ and CZ in the $300 \mathrm{~ms}$ prior to the onset of the stimulus array (see Fig. 2). This revealed a main effect of Condition, $F(1$, $40)=6.11, p=.018, \eta_{\mathrm{p}}{ }^{2}=.13$, such that the SPN was more negative for standard $(M=-2.13, S D=3.31)$ than self-focus trials $(M=-1.41, S D=1.96 ; d=.27)$. The main effect of Group was not significant, nor was the interaction, $F \mathrm{~s}<1.0$.

\section{Performance}

Response patterns indicated that no participant engaged in invariant responding, and response rates did not significantly differ as a function of target type, whether self-focus cues were present, or whether target array stimuli changed. There was a main effect of Target, $F(1,40)=59.76, p<.001, \eta_{\mathrm{p}}^{2}=.60$, such that performance was better when disgust faces occupied the target hemifield $(M=.57, S D=.05)$ rather than neutral faces $(M=.51, S D=.06)$. There was a nonsignificant trend, $F(1,40)=3.15, p=.08, \eta_{\mathrm{p}}^{2}=.07$, toward impaired performance when self-focus cues were present $(M=$ $.53, S D=.06)$ compared to standard cues $(M=.55$, $S D=.06)$. There were no significant interactions or group effects.

\section{Primary analyses ${ }^{1}$}

Analyses of the N2pc for disgust faces revealed a significant three-way interaction of Group, Condition, and Laterality, $F(1,40)=7.72, p<.01, \eta_{\mathrm{p}}^{2}=.16$, such that HSA subjects showed greater negativity at sites contralateral $(M=2.03, S D=3.85)$ than sites ipsilateral to disgust faces for standard trials $(M=2.20, S D=3.88$; $\left.p<.01, \eta_{\mathrm{p}}^{2}=.16\right)$, but not self-focus trials, $p=.24$. Thus, there was a significant N2pc for disgust faces for HSAs on standard trials. In contrast, LSA subjects showed marginally significant negativity at contralateral $(M=1.75, S D=3.95)$ relative to ipsilateral sites $(M=$ $\left.1.88, S D=3.94, p=.06, \eta_{\mathrm{p}}^{2}=.08\right)$ for disgust faces on self-focus trials but not standard trials, $p=.76$ (see Figs. 3 and 4), suggesting an N2pc for disgust faces only during presentation of self-focus cues. There were no other significant main effects or interactions.

For the uncued CDA, there was a significant three-way interaction of Group, Condition, and Laterality, $F(1,40)=$ $5.43, p=.025, \eta_{\mathrm{p}}^{2}=.12$ (see Figs. 3 and 4). Follow-up pairwise comparisons indicated that on standard trials, HSAs showed greater contralateral $(M=.66, S D=2.38)$ than ipsilateral negativity $(M=.84, S D=2.40)$ for disgust faces, $p<.01, \eta_{\mathrm{p}}{ }^{2}=$ .16 , but this effect was not present for trials with self-focus cues, $p=.16$, nor was it significant for LSA subjects for trials with self-focus cues, $p=.53$, or without them, $p=.96$. Thus, HSAs had a significant CDA for disgust faces on standard, but not self-focus, trials, and LSAs showed no CDA. No other effects were significant.

Analysis of the cued CDA (see Fig. 5) revealed a marginally significant main effect for Laterality, $F(1,40)=3.93, p=$ $.054, \eta_{\mathrm{p}}{ }^{2}=.09$. This was qualified by an interaction between Group and Laterality, $F(1,40)=5.11, p=.029, \eta_{\mathrm{p}}{ }^{2}=.11$. Unexpectedly, LSA subjects showed greater negativity at sites ipsilateral to targets $(M=.80, S D=2.15)$ than at contralateral sites $\left(M=1.09, S D=2.27 ; p=.004, \eta_{\mathrm{p}}{ }^{2}=.19\right)$. No significant pairwise comparisons were found for the HSA group, nor were there other significant main effects or interactions.

\section{Supplementary analysis}

Further analysis of the ERP waveform following the target cue was conducted due to the unexpected reverse direction of the cued CDA and a visual inspection of

\footnotetext{
${ }^{1}$ All primary analyses were reanalyzed while controlling for depression symptoms (i.e., BDI-II score). All analyses remained significant with the exception of the three-way interaction for the uncued CDA, which was marginally significant, $F(1,39)=3.76, p=.06$. The primary analyses were repeated with SIAS score as a continuous variable rather than using extreme groups. The three-way interaction for the N2pc remained significant, $F(1,40)=5.25, p=.027, \eta_{\mathrm{p}}^{2}=.12$, and the interaction for the uncued CDA was marginal, $F(1,40)=4.04, p=.051, \eta_{\mathrm{p}}^{2}=.09$.
} 


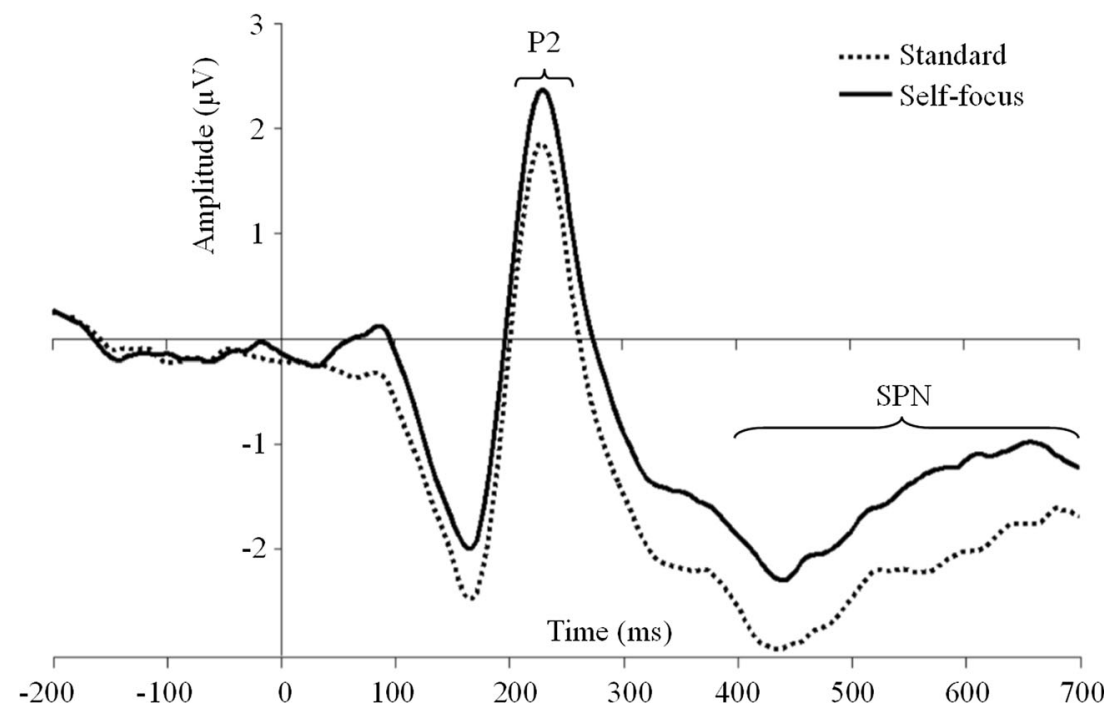

Fig. 2 P2 and SPN event locked to fixation onset

the waveforms that suggested that this reverse effect began around $400 \mathrm{~ms}$. To verify that subjects followed task instructions to attend to the target hemifield, the early part of the cued CDA (i.e., mean voltage from 200-300 ms post cue) was examined. Analysis of this early cued CDA revealed a main effect for Laterality, $F(1,40)=5.98, p=.02, \eta_{\mathrm{p}}{ }^{2}=.13$, such that subjects showed greater negativity at sites contralateral $(M=$ $2.57, S D=2.29)$ than sites ipsilateral to targets $(M=$ $2.70, S D=2.34)$. Thus, subjects showed evidence of early preferential maintenance for the target hemifield. There were no other significant main effects or interactions.

\section{Discussion}

The goal of this study was to use lateralized ERPs to evaluate discrepant theoretical predictions with respect to attentional biases associated with social anxiety. The key findings

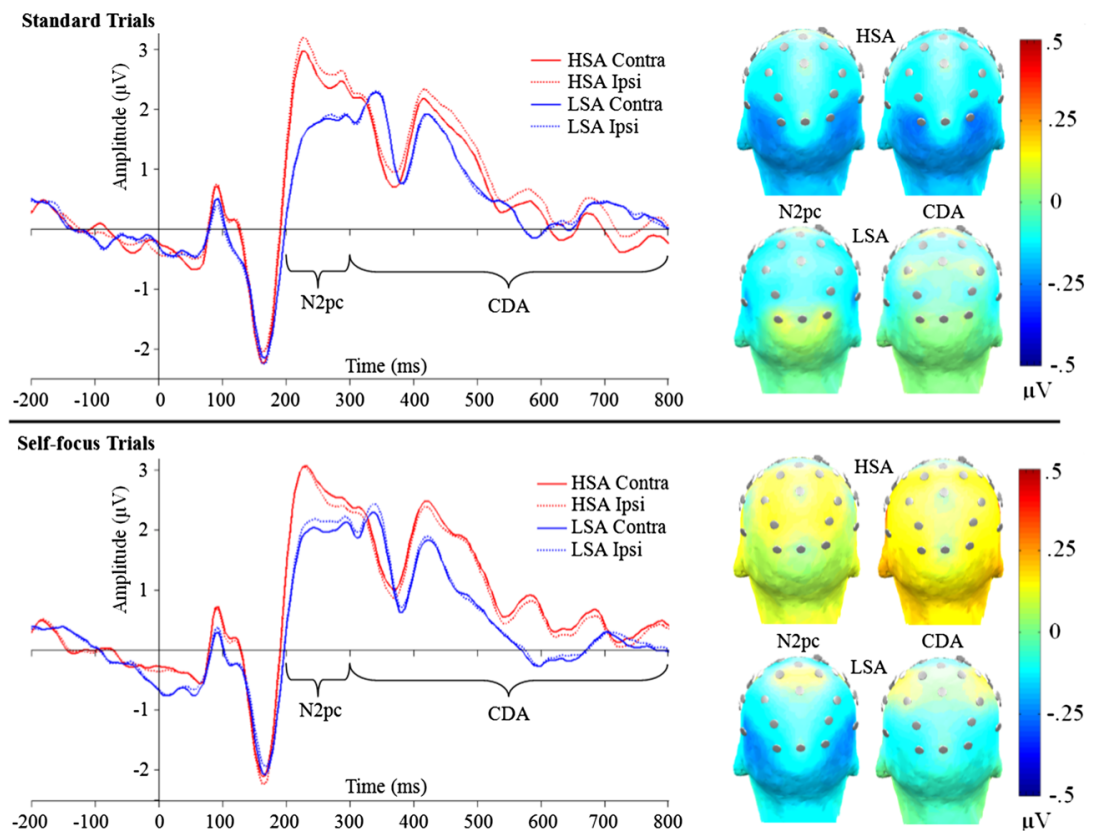

Note: Contralateral and ipsilateral waveforms are relative to disgust faces. Scalp maps display the mean voltage difference (contra minus ipsi) within the measurement windows.

Fig. 3 N2pc and uncued CDA event locked to stimulus array onset 


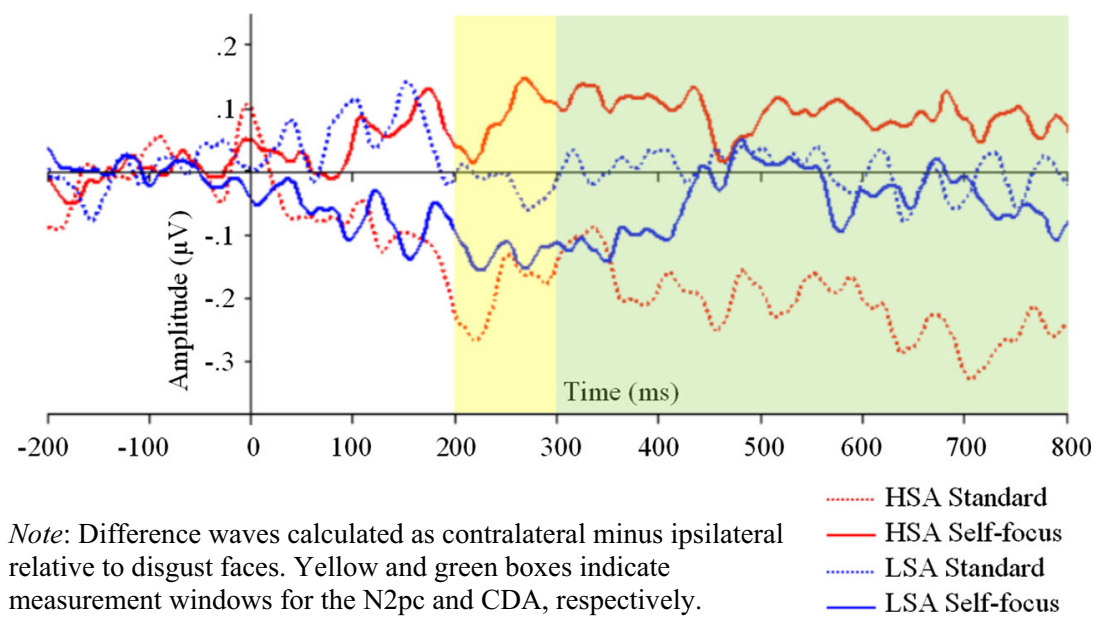

Fig. 4 N2pc and uncued CDA difference waves. (Color figure online.)

suggested that selective attention for self-focus cues was correlated with social anxiety and correlated with reduced performance in socially anxious subjects. Socially anxious individuals showed a selection bias for disgust faces, and this bias persisted in working memory. However, both biases were not observed during presentation of self-focus cues.

A notable finding of this study is that socially anxious subjects show biases in selective attention (N2pc) and working memory maintenance (uncued CDA) for disgust relative to neutral faces and that individuals low in social anxiety do not. This supports cognitive theories of SAD and research on early attentional biases (e.g., Mueller et al., 2009). The current study advances this literature by examining these biases with concurrent neutral and disgust faces. Additionally, the uncued CDA findings suggest that socially anxious individuals preferentially maintain disgust faces in working memory even after they are no longer present. Previous social anxiety research has found delays in ERPs related to the categorization of faces that follow disgust faces, perhaps suggesting the involuntary persistence of disgust faces in working memory (Rossignol, Anselme, Vermeulen, Philippot, \& Campanella, 2007). This sustained bias in working memory may be relevant to the ongoing question of the conditions in which social threat stimuli are attended or disengaged at late stages of processing. Notably, equivalent scores on the letter-number sequencing task suggests that differences in the CDA were not due to differences in working memory capacity between the groups.

In support of the self-focus manipulation, subjects reported that the self-focus cues led to greater self-consciousness and interfered with performance. There was a nonsignificant trend toward actual performance interference when self-focus cues were present $(p=.08)$. Subjects showed greater selective attention (i.e., anterior P2 amplitude) for self-focus than for standard cues. This attention to self-focus cues was correlated with reduced performance for socially anxious subjects, but

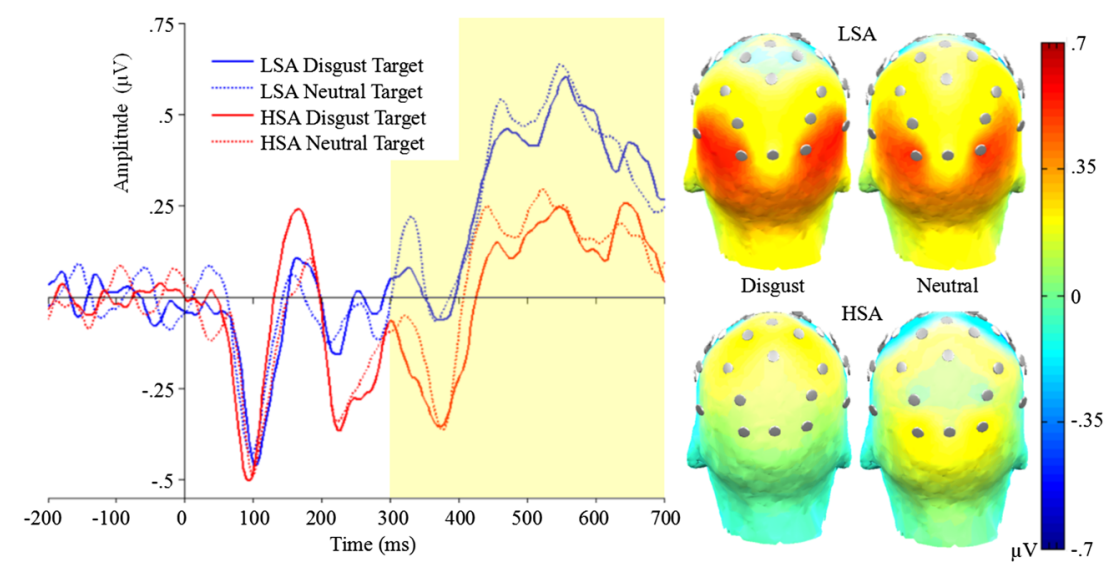

Note: Difference waves calculated as contralateral minus ipsilateral relative to target hemifield. Yellow box indicates measurement window.

Fig. 5 Cued CDA difference waves event locked to target arrow onset. (Color figure online.) 
not those low in social anxiety. Subjects also displayed an attenuation of the SPN preceding stimulus arrays when selffocus cues were present, suggesting that self-focus cues reduced anticipatory resources for processing of upcoming facial stimuli. Consistent with this, socially anxious individuals did not display N2pc or CDA effects for disgust stimuli following self-focus cues. This is partially in line with previous research that found that similar cues attenuated the SPN in socially anxious subjects (Judah, Grant, Mills, et al., 2013). It also supports cognitive models, which suggest that selffocus can reduce the availability of attention for external stimuli (e.g., Clark \& Wells, 1995).

Although there was not a significant difference in P2 amplitude between groups, the difference in P2 amplitude for self-focus and standard cues was correlated with social anxiety when controlling for depression. This suggests that the portion of the P2 indicating physiological sensitivity is related to social anxiety but may be masked by depression. It will be important for future studies to consider how depression may affect relationships between social anxiety and measures of self-focused attention. The results suggest that the consequences of self-focus are different between those with high and low levels of social anxiety, as evidenced by the betweengroups effects of self-focus on the N2pc and CDA. Research is needed to further investigate how self-focus may deplete attentional resources in socially anxious individuals and alter the allocation of attention to external stimuli. The self-focus results are partially inconsistent with a previous study that found that socially anxious individuals, compared to those low in social anxiety, reported greater self-consciousness and task interference from self-focus cues (Judah et al., 2013). The previous study presented heart rate feedback on $20 \%$ of trials, whereas it was presented on $50 \%$ of trials in this study to avoid eliciting an oddball effect. More research is needed to determine how self-focused attention is affected by the frequency and duration of heart-rate feedback.

Although the current study supports a sustained bias for social threat, it is unclear whether changes to the experimental design might have produced different results. For example, Judah, Grant, Lechner, et al. ( 2013) found that under cognitive load, socially anxious individuals showed sustained attention to disgust faces, but low load resulted in attentional disengagement from these faces. It is possible that the current task, which required participants to store features of four faces simultaneously, resulted in high cognitive load, thereby depleting executive resources that might be used to disengage attention from threatening stimuli if this load were not present. Previous work has shown that individual differences in the CDA may arise from inefficient use of cognitive resources (e.g., Vogel et al., 2005), and that anxious individuals may fail to prevent storage of task-irrelevant, threat-related stimuli (Stout et al., 2013). Thus, it is possible that the use of socially threatening stimuli resulted in differential load for the groups.
Further research is needed to investigate this and other potential moderators of the relationship between social anxiety and working memory biases.

These data also suggest interesting effects of self-focus on attentional biases. There is a lack of clear consensus among theories (see Schultz \& Heimberg, 2008) as to whether selffocused attention increases the salience of external social threat stimuli and thereby increases vigilance (Heimberg et al., 2010) or reduces the availability of attentional resources to process external stimuli (Clark \& McManus, 2002). This study supported the latter by finding that self-focus resulted in a lack of bias for disgust faces among socially anxious individuals.

Unexpectedly, the self-focus manipulation resulted in a marginally significant bias in selective attention (i.e., N2pc) for disgust faces among subjects low in social anxiety. This suggests a differential effect of the manipulation on attentional biases among individuals high and low in social anxiety. Whereas it resulted in reduced bias for the socially anxious subjects, it increased this bias in those with low social anxiety. Subjects low in social anxiety did not show maintenance of this bias in working memory, even for self-focus trials. This may suggest a mechanism for the development of social anxiety. Cues of physiological arousal may prompt brief vigilance for social threat in nonanxious individuals. Future research is needed to determine whether this effect is replicable, particularly in light of its marginal significance.

In contrast to expectations, a cued CDA was not evident in this study. It was hypothesized that subjects would show greater negativity at contralateral compared to ipsilateral sites for targets in the interval preceding the test array. Counter to this hypothesis, the low social anxiety group showed the opposite pattern, namely, greater negativity at ipsilateral than contralateral sites. Analysis of the early cued CDA suggested that both groups showed early selective maintenance in favor of the target hemifield, ruling out the possibility that subjects were not following target cues. One possibility is that the ipsilateral negativity indicates processing of stimuli in the nontarget hemifield (see Arend \& Zimmer, 2011). Alternatively, the difficulty of the task or the appearance of target hemifield cues after the stimulus array may have played a role in this unusual effect. These possibilities are speculative, and clear conclusions cannot be drawn at this time. More research is needed to clarify the nature of the ipsilateral delay activity.

There are a number of limitations to this study. First, the use of a nonclinical, undergraduate sample limits the generalizability of the findings. However, recent perspectives on psychopathology (e.g., Insel et al., 2010) suggest that research is needed that targets symptoms, behavior, and biology rather than diagnostic categories. Many studies, including this one, have not compared biases across disorders. As such, it is unclear whether the effects observed are specific to social 
anxiety. Future studies using clinical samples with a variety of anxiety disorders are needed. The use of the SIAS to operationalize social anxiety is consistent with many previous studies, but different results may have been obtained had a measure of social scrutiny fears or fear of negative evaluation been used. The low performance rates in this study may have been due to the brief presentation of facial stimuli (i.e., 200 $\mathrm{ms}$ ), the long storage interval (i.e., 1,500 ms), the presentation of the target cue after the stimulus array rather than before it, and/or the need to detect changes in face identities rather than a simple feature, such as face gender. Although there was a high response rate, this does not necessarily support engagement to the task. However, the ERP findings support processing of the stimuli, and the key findings of the study are not dependent upon task performance.

The cued CDA amplitudes showed slight negative skew and kurtosis in some conditions. Although ANOVA is relatively robust against violations of the normality assumption, this limits the conclusions that can be drawn from these findings. A few studies suggest that subjects with SAD may find neutral faces to be threatening (e.g., Cooney, Atlas, Joormann, Eugène, \& Gotlib, 2006; Lange, Allart, Keijsers, Rinck, \& Becker, 2012). Future studies are needed to examine whether socially anxious individuals display greater processing of neutral faces and/or interpret them as more threatening compared to healthy controls, even while displaying a particular bias for disgust faces, as noted in our data. Future studies may avoid describing the self-focus symbol as a glitch to avoid this as a rival explanation for the effects. The effects of the self-focus manipulation in this study should be considered preliminary, and further validation is needed in future studies. Additional studies are needed that utilize social manipulations, a between-groups design, or a changing cue that does not convey physiological information as a control.

This study has several important implications. It supports cognitive models of SAD by providing evidence of attentional biases for social threat. The data address a long-standing distinction between theories regarding the effect of self-focused attention on external attention to threatening stimuli. New research questions are suggested by the results, and these may be useful for honing cognitive theories. In addition to the theoretical implications of the study, the findings may be important for treatment considerations. Cognitive behavioral therapy (CBT) for $\mathrm{SAD}$, which relies on exposure therapy, may benefit from this and other studies that are progressively explicating how attentional biases maintain social fears. For example, these data may be taken as part of a building literature suggesting that self-focused attention may reduce processing of threat in the environment, which may impact the success of exposure. More research is needed to further our understanding of internal and external attentional biases and how this knowledge can advance treatments.
Acknowledgments This work was supported by Oklahoma State University [Robberson Dissertation Fellowship; Distinguished Graduate Fellowship]; and the American Psychological Foundation/Council of Graduate Departments of Psychology [Graduate Research Scholarship in Psychology]. The views expressed in this article are those of the authors and do not necessarily reflect the position or policy of Oklahoma State University, the American Psychological Foundation, or the Council of Graduate Departments of Psychology. All authors report no actual or potential conflicts of interest.

\section{References}

Amir, N., \& Bomyea, J. (2011). Working memory capacity in generalized social phobia. Journal of Abnormal Psychology, 120, 504-509. doi: 10.1037/a0022849

Amir, N., Freshman, M., \& Foa, E. (2002). Enhanced Stroop interference for threat in social phobia. Journal of Anxiety Disorders, 16, 1-9. doi:10.1016/S0887-6185(01)

Amir, N., Klumpp, H., Elias, J., Bedwell, J. S., Yanasak, N., \& Miller, L. S. (2005). Increased activation of the anterior cingulate cortex during processing of disgust faces in individuals with social phobia. Biological Psychiatry, 57, 975-981. doi:10.1016/j.biopsych.2005. 01.044

Amir, N., Najmi, S., Bomyea, J., \& Burns, M. (2010). Disgust and anger in social anxiety [Special section: Disgust and psychopathology]. International Journal of Cognitive Therapy, 3, 3-10. doi:10.1521/ ijct.2010.3.1.3

Anllo-Vento, L., Luck, S. J., \& Hillyard, S. A. (1998). Spatio-termporal dynamics of attention to color: Evidence from human electrophysiology. Human Brain Mapping, 6, 216-238. doi:10.1002/(SICI) 1097-0193(1998)6:4<216::AID-HBM3>3.0.CO;2-6

Arend, A. M., \& Zimmer, H. D. (2011). What does ipsilateral delay activity reflect? Inferences from slow potentials in a lateralized visual working memory task. Journal of Cognitive Neuroscience, 23, 4048-4056. doi:10.1162/jocn_a_00068

Beck, A. T., Steer, R. A., \& Brown, G. K. (1996). Manual for Beck Depression Inventory-II. San Antonio: Psychological Corporation.

Buckner, J. D., Maner, J. K., \& Schmidt, N. B. (2010). Difficulty disengaging attention from social threat in social anxiety. Cognitive Therapy and Research, 34, 99-105. doi:10.1007/ s10608-008-9205-y

Clark, D. M., \& McManus, F. (2002). Information processing in social phobia. Biological Psychiatry, 51, 92-100. doi:10.1016/S00063223(01)

Clark, D. M., \& Wells, A. (1995). A cognitive model of social phobia. In R. Heimberg, M. Liebowitz, D. A. Hope, \& F. R. Schneier (Eds.), Social phobia: Diagnosis, assessment, and treatment (pp. 69-93). New York: Guilford Press.

Cohen, J. (1988). Statistical power analysis for the behavioral science. Hillsdale: Erlbaum.

Cooney, R. E., Atlas, L. Y., Joormann, J., Eugène, F., \& Gotlib, I. H. (2006). Amygdala activation in the processing of neutral faces in social anxiety disorder: Is neutral really neutral? Psychiatry Research: Neuroimaging, 148, 55-59. doi:10.1016/j.pscychresns. 2006.05.003

Delorme, A., \& Makeig, S. (2004). EEGLAB: An open source toolbox for analysis of single-trial EEG dynamics. Journal of Neuroscience Methods, 134, 9-21. doi:10.1016/j.jneumeth.2003.10.009

Domschke, K., Stevens, S., Pfleiderer, B., \& Gerlach, A. L. (2010). Interoceptive sensitivity in anxiety and anxiety disorders: An overview and integration of neurobiological findings. Clinical Psychology Review, 30, 1-11. doi:10.1016/j.cpr.2009.08.008 
Eimer, M., \& Kiss, M. (2007). Attentional capture by task-irrelevant fearful faces is revealed by the N2pc component. Biological Psychology, 74, 108-112. doi:10.1016/j.biopsycho.2006.06.008

Gilboa-Schechtman, E., Foa, E. B., \& Amir, N. (1999). Attentional biases for facial expressions in social phobia: The face-in-the-crowd paradigm. Cognition \& Emotion, 13, 305-318. doi:10.1080/ 026999399379294

Grant, D. M., \& Beck, J. G. (2006). Attentional biases in social anxiety and dysphoria: Does comorbidity make a difference? Journal of Anxiety Disorders, 20, 520-529. doi:10.1016/j.janxdis.2005.05.003

Grant, D. M., Judah, M. R., White, E. J., \& Mills, A. C. (2015). Worry and discrimination of threat and safety cues: An event-related potential investigation. Behavior Therapy, 46(5), 652-660. doi:10. 1016/j.beth.2014.09.015

Heimberg, R. G., Brozovich, F. A., \& Rapee, R. M. (2010). A cognitivebehavioral model of social anxiety disorder: Update and extension. In S. G. Hofmann \& P. M. DiBartolo (Eds.), Social anxiety: Clinical, developmental, and social perspectives (2nd ed., pp. 395-422). New York, NY: Academic Press. doi:10.1016/B978-0-12-375096-9. 00015-8

Insel, T., Cuthbert, B., Garvey, M., Heinssen, R., Pine, D. S., Quinn, K., ...Wang, P. (2010). Research domain criteria (RDoC): Toward a new classification framework for research on mental disorders. American Journal of Psychiatry, 167, 748-751. doi:10.1176/appi. ajp.2010.09091379

Judah, M. R., Grant, D. M., Lechner, W. V., \& Mills, A. C. (2013a). Working memory load moderates late attentional bias in social anxiety. Cognition \& Emotion, 27, 502-511. doi:10.1080/02699931. 2012.719490

Judah, M. R., Grant, D. M., Mills, A. C., \& Lechner, W. V. (2013b). The neural correlates of impaired attentional control in social anxiety: An ERP study of inhibition and shifting. Emotion. doi:10.1037/ a0033531

Juth, P., Lundqvist, D., Karlsson, A., \& Öhman, A. (2005). Looking for foes and friends: Perceptual and emotional factors when finding a face in the crowd. Emotion, 5, 379-395. doi:10.1037/1528-3542.5. 4.379

Kappenman, E. S., Farrens, J. L., Luck, S. J., \& Proudfit, G. H. (2014). Behavioral and ERP measures of attentional bias to threat in the dotprobe task: Poor reliability and lack of correlation with anxiety. Frontiers in Psychology, 5(1368), 1-9. doi:10.3389/fpsyg.2014. 01368

Kiss, M., Van Velzen, J., \& Eimer, M. (2008). The N2pc component and its links to attention shifts and spatially selective visual processing. Psychophysiology, 45, 240-249. doi:10.1111/j.1469-8986.2007. 00611.x

Kolassa, I.-T., Kolassa, S., Bergmann, S., Lauche, R., Dilger, S., Miltner, W. H. R., \& Musial, F. (2009). Interpretive bias in social phobia: An ERP study with morphed emotional schematic faces. Cognition and Emotion, 23, 69-95. doi:10.1080/02699930801940461

Kolassa, I.-T., Kolassa, S., Musial, F., \& Miltner, W. H. R. (2007). Eventrelated potentials to schematic faces in social phobia. Cognition \& Emotion, 21, 1721-1744. doi:10.1080/02699930701229189

Lange, W.-G., Allart, E., Keijsers, G. P. J., Rinck, M., \& Becker, E. S. (2012). A neutral face is not neutral even if you have not seen it: Social anxiety disorder and affective priming with facial expressions. Cognitive Behaviour Therapy, 41, 108-118. doi:10.1080/ 16506073.2012.666563

Langner, O., Dotsch, R., Bijlstra, G., Wigboldus, D. H. J., Hawk, S. T., \& Van Knippenberg, A. (2010). Presentation and validation of the Radboud Faces Database. Cognition \& Emotion, 24, 1377-1388. doi:10.1080/02699930903485076

LeMoult, J., \& Joormann, J. (2012). Attention and memory biases in social anxiety disorder: The role of comorbid depression. Cognitive Therapy and Research, 36, 47-57. doi:10.1007/s10608010-9322-2
Lopez-Calderon, J., \& Luck, S. J. (2013). The ERPLAB toolbox (Version 4.0) Retrieved from http://erpinfo.org/erplab

Luck, S. J., \& Hillyard, S. A. (1994). Electrophysiological correlates of feature analysis during visual search. Psychophysiology, 31, 291308. doi:10.1111/j.1469-8986.1994.tb02218.x

Mansell, W., \& Clark, D. M. (1999). How do I appear to others? Social anxiety and processing of the observable self. Behaviour Research and Therapy, 37, 419-434. doi:10.1016/S0005-7967(98)00148-X

Mansell, W., Clark, D. M., \& Ehlers, A. (2003). Internal versus external attention in social anxiety: An investigation using a novel paradigm. Behaviour Research and Therapy, 41, 555-572. doi:10.1016/ S0005-7967(02)00029-3

Mattick, R. P., \& Clarke, J. C. (1989). Development and validation of measures of social phobia scrutiny fear and social interaction anxiety. Behaviour Research and Therapy, 36, 455-470. doi:10.1016/ S0005-7967(97)10031-6

McTeague, L. M., Shumen, J. R., Wieser, M. J., Lang, P. J., \& Keil, A. (2011). Social vision: Sustained perceptual enhancement of affective facial cues in social anxiety. NeuroImage, 54, 1615-1624. doi:10. 1016/j.neuroimage.2010.08.080

Mellings, T. B., \& Alden, L. E. (2000). Cognitive processes in social anxiety: The effects of self-focus, rumination and anticipatory processing. Behaviour Research and Therapy, 38, 243-257. doi:10. 1016/S0005-7967(99)00040-6

Mills, A. C., Grant, D. M., Judah, M. R., \& White, E. J. (2014). The influence of anticipatory processing on attentional biases in social anxiety. Behavior Therapy, 45, 720-729. doi:10.1016/j.beth.2014. 04.004

Mogg, K., Philippot, P., \& Bradley, B. P. (2004). Selective attention to angry faces in clinical social phobia. Journal of Abnormal Psychology, 113, 160-165. doi:10.1037/0021-843X.113.1.160

Mognon, A., Jovicich, J., Bruzzone, L., \& Buiatti, M. (2011). ADJUST: An automatic EEG artifact detector based on the joint use of spatial and temporal features. Psychophysiology, 48, 229-240. doi:10.1111/ j.1469-8986.2010.01061.x

Moriya, J., \& Sugiura, Y. (2012a). High visual working memory capacity in trait social anxiety. PLOS ONE, 7(e34244), 1-6. doi:10.1371/ journal.pone.0034244

Moriya, J., \& Sugiura, Y. (2012b). Impaired attentional disengagement from stimuli matching the contents of working memory in social anxiety. PLOS ONE, 7(e47221), 1-8. doi:10.1371/journal.pone. 0047221

Moriya, J., \& Sugiura, Y. (2013). Socially anxious individuals with low working memory capacity could not inhibit the goal-irrelevant information. Frontiers in Human Neuroscience, 7(840), 1-8. doi:10. 3389/fnhum.2013.00840

Moser, J. S., Huppert, J. D., Duval, E., \& Simons, R. F. (2008). Face processing biases in social anxiety: An electrophysiological study. Biological Psychology, 78, 93-103. doi:10.1016/j.biopsycho.2008. 01.005

Mueller, E. M., Hofmann, S. G., Santesso, D. L., Meuret, A. E., Bitran, S. S., \& Pizzagalli, D. A. (2009). Electrophysiological evidence of attentional biases in social anxiety disorder. Psychological Medicine: A Journal of Research in Psychiatry and the Allied Sciences, 39, 1141-1152. doi:10.1017/S0033291708004820

Mühlberger, A., Wieser, M. J., Herrmann, M. J., Weyers, P., Tröger, C., \& Pauli, P. (2009). Early cortical processing of natural and artificial emotional faces differs between lower and higher socially anxious persons. Journal of Neural Transmission, 116, 735-746. doi:10. 1007/s00702-008-0108-6

Papageorgiou, C., \& Wells, A. (2002). Effects of heart rate information on anxiety, perspective taking, and performance in high and low socialevaluative anxiety. Behavior Therapy, 33, 181-199. doi:10.1016/ S0005-7894(02)80024-7 
Peirce, J. W. (2007). PsychoPy: Psychophysics software in Python. Journal of Neuroscience Methods, 162, 8-13. doi:10.1016/j. jneumeth.2006.11.017

Peschard, V., Philippot, P., Joassin, F., \& Rossignol, M. (2013). The impact of the stimulus features and task instructions on facial processing in social anxiety: An ERP investigation. Biological Psychology, 93, 88-96. doi:10.1016/j.biopsycho.2013.01.009

Pini, S., Cassano, G. B., Simonini, E., Savino, M., Russo, A., \& Montgomery, S. A. (1997). Prevalence of anxiety disorders comorbidity in bipolar depression, unipolar depression and dysthymia. Journal of Affective Disorders, 42, 145-153. doi:10.1016/S01650327(96)01405-X

Potts, G. F. (2004). An ERP index of task relevance evaluation of visual stimuli. Brain and Cognition, 56, 5-13. doi:10.1016/j.bandc.2004. 03.006

Rapee, R. M., \& Heimberg, R. G. (1997). A cognitive-behavioral model of anxiety in social phobia. Behaviour Research and Therapy, 35, 741-756. doi:10.1016/S0005-7967(97)00022-3

Rossignol, M., Anselme, C., Vermeulen, N., Philippot, P., \& Campanella, S. (2007). Categorical perception of anger and disgust facial expression is affected by non-clinical social anxiety: An ERP study. Brain Research, 1132, 166-176. doi:10.1016/j.brainres.2006.11.036

Rossignol, M., Campanella, S., Maurage, P., Heeren, A., Falbo, L., \& Philippot, P. (2012a). Enhanced perceptual responses during visual processing of facial stimuli in young socially anxious individuals. Neuroscience Letters, 526, 68-73. doi:10.1016/j.neulet.2012.07. 045

Rossignol, M., Philippot, P., Bissot, C., Rigoulot, S., \& Campanella, S. (2012b). Electrophysiological correlates of enhanced perceptual processes and attentional capture by emotional faces in social anxiety. Brain Research, 1460, 50-62. doi:10.1016/j.brainres.2012.04. 034

Rossignol, M., Campanella, S., Bissot, C., \& Philippot, P. (2013). Fear of negative evaluation and attentional bias for facial expressions: An event-related study. Brain and Cognition, 82, 344-352. doi:10. 1016/j.bandc. 2013.05 .008

Schofield, C. A., Johnson, A. L., Inhoff, A. W., \& Coles, M. E. (2012). Social anxiety and difficulty disengaging threat: Evidence from eyetracking. Cognition and Emotion, 26, 300-311. doi:10.1080/ 02699931.2011.602050

Schultz, L. T., \& Heimberg, R. G. (2008). Attentional focus in social anxiety disorder: Potential for interactive processes. Clinical Psychology Review, 28, 1206-1221. doi:10.1016/j.cpr.2008.04.003

Segal, A., Kessler, Y., \& Anholt, G. E. (2015). Updating the emotional content of working memory in social anxiety. Journal of Behavior Therapy and Experimental Psychiatry, 48, 110-117. doi:10.1016/j. jbtep.2015.02.012

Sewell, C., Palermo, R., Atkinson, C., \& McArthur, G. (2008). Anxiety and neural processing of threat in faces. NeuroReport, 19, 13391343. doi:10.1097/WNR.0b013e32830baadf

Singh, J. S., Capozzoli, M. C., Dodd, M. D., \& Hope, D. A. (2015). The effects of social anxiety and state anxiety on visual attention: Testing the vigilance-avoidance hypothesis. Cognitive Behaviour Therapy, 44(5), 377-388. doi:10.1080/16506073.2015.1016447

Sposari, J. A., \& Rapee, R. M. (2007). Attentional bias toward facial stimuli under conditions of social threat in socially phobic and nonclinical participants. Cognitive Therapy and Research, 31, 23-37. doi:10.1007/s10608-006-9073-2

Spurr, J. M., \& Stopa, L. (2002). Self-focused attention in social phobia and social anxiety. Clinical Psychology Review, 22, 947-975. doi: 10.1016/S0272-7358(02)00107-1

Staugaard, S. R. (2010). Threatening faces and social anxiety: A literature review. Clinical Psychology Review, 30, 669-690. doi:10.1016/j. cpr.2010.05.001

Storch, E. A., Roberti, J. W., \& Roth, D. A. (2004). Factor structure, concurrent, validity, and internal consistency of the Beck Depression Inventory-Second Edition in a sample of college students. Depression and Anxiety, 19, 187-189. doi:10.1002/da.20002

Stout, D. M., Shackman, A. J., \& Larson, C. L. (2013). Failure to filter: Anxious individuals show inefficient gating of threat from working memory. Frontiers in Human Neuroscience, 7(58), 1-10. doi:10. 3389/fnhum.2013.00058

Van Boxtel, G. J. M., \& Böcker, K. B. E. (2004). Cortical measures of anticipation. Journal of Psychophysiology, 18, 61-71. doi:10.1027/ 0269-8803.18.23.61

Van Peer, J. M., Spinhoven, P., \& Roelofs, K. (2010). Psychophysiological evidence for cortisol-induced reduction in early bias for implicit social threat in social phobia. Psychoneuroendocrinology, 35, 21-32. doi:10.1016/j.psyneuen. 2009.09.012

Vogel, E. K., \& Machizawa, M. G. (2004). Neural activity predicts individual differences in visual working memory. Nature, 428, 748-751. doi:10.1038/nature02447

Vogel, E. K., McCollough, A. W., \& Machizawa, M. G. (2005). Neural measures reveal individual differences in controlling access to working memory. Nature, 438, 500-503. doi:10.1038/nature04171

Wechsler, D. (1997). WAIS III: Wechsler Adult Intelligence Scale (3rd ed.). San Antonio: The Psychological Corporation.

Wells, A., \& Papageorgiou, C. (2001). Social phobic interoception: Effects of bodily information on anxiety, beliefs and self-processing. Behaviour Research and Therapy, 39, 1-11. doi:10.1016/S00057967(99)00146-1

Weymar, M., Gerdes, A. B. M., Löw, A., Alpers, G. W., \& Hamm, A. O. (2013). Specific fear modulates attentional selectivity during visual search: Electrophysiological insights from the N2pc. Psychophysiology, 50, 139-148. doi:10.1111/psyp.12008

Wild, J., Clark, D. M., Ehlers, A., \& McManus, F. (2008). Perception of arousal in social anxiety: Effects of false feedback during a social interaction. Behavior Therapy and Experimental Psychiatry, 39, 102-116. doi:10.1016/j.jbtep.2006.11.003

Woodman, G. F., \& Vogel, E. K. (2008). Selective storage and maintenance of an object's features in visual working memory. Psychonomic Bulletin \& Review, 15, 223-229. doi:10.3758/PBR. 15.1.223 